\title{
Re-gripping analysis based on implementation of slip-detection device for robotic hand model
}

\begin{abstract}
To develop an intelligent robotic hand, diverse approaches have been applied, including optimum gripping force and slippage analysis. In this study, a robotic hand was modeled with tactile pressure sensors. The slip detection sensor, which is represented as a rotary encoder device, was employed to indicate the slip situation features, distance and velocity. Empirical findings imply that the correlation between the distance that an object has slipped and the required re-gripping force was developed to be availed as an automatic feedback algorithm. The slippage events were revealed and analyzed to perform the control system mechanism of the re-gripping mission.
\end{abstract}

Keyword: Automatic feedback algorithm; Re-gripping operation; Robotic hand model; Slipdetection sensor 